\title{
Newton's Hyperbola Observed from Newton's Evolute (1687), Gudermann's Circle (1833), the Auxiliary Circle (Pedal Curve and Inversion Curve), the Lemniscate of Bernoulli (1694) (Pedal Curve and Inversion Curve) (09.01.2019)
}

\author{
Jiří Stávek ${ }^{1}$ \\ ${ }^{1}$ Bazovského, Prague, Czech Republic \\ Correspondence: Jiř́i Stávek, Bazovského 1228, 16300 Prague, Czech Republic. E-mail: stavek.jiri@seznam.cz
}

Received: January 5, 2019

Accepted: January 21, 2019

Online Published: January 29, 2019

doi:10.5539/apr.v11n1p65

URL: http://dx.doi.org/10.5539/apr.v11n1p65

\begin{abstract}
Johannes Kepler and Isaac Newton inspired generations of researchers to study properties of elliptic, hyperbolic, and parabolic paths of planets orbiting around the Sun. After the intensive study of those conic sections during the last four hundred years it is believed that this topic is practically closed and the $21^{\text {st }}$ Century cannot bring anything new to this subject. Can we add to those visible orbits from the Aristotelian World some curves from the Plato's Realm that might bring to us new information about those conic sections? Isaac Newton in 1687 discovered one such curve - the evolute of the hyperbola - behind his famous gravitation law. In our model we have been working with Newton's Hyperbola in a more complex way. We have found that the interplay of the empty focus M (= Menaechmus - the discoverer of hyperbola), the center of the hyperbola A (= Apollonius of Perga - the Great Geometer), and the occupied focus N (= Isaac Newton - the Great Mathematician) together form the MAN Hyperbola with several interesting hidden properties of those hyperbolic paths. We have found that the auxiliary circle of the MAN Hyperbola could be used as a new hodograph and we will get the tangent velocity of planets around the Sun and their moment of tangent momentum. We can use the lemniscate of Bernoulli as the pedal curve of that hyperbola and we will get the normal velocities of those orbiting planets and their moment of normal momentum. The first derivation of this moment of normal momentum will reveal the torque of that hyperbola and we can estimate the precession of hyperbolic paths and to test this model for the case of the flyby anomalies. The auxiliary circle might be used as the inversion curve of that hyperbola and the Lemniscate of Bernoulli could help us to describe the Kepler's Equation (KE) for the hyperbolic paths. Have we found the Arriadne's Thread leading out of the Labyrinth or are we still lost in the Labyrinth?
\end{abstract}

Keywords: Newton's Hyperbola, Aristotelian World, Plato's Realm, Hidden Mathematical Objects, MAN Hyperbola, Newton's Evolute, Gudermann's Circle, Pedal Curves, Inversion Curves, Torque of Ellipse, Kepler's Equation for Hyperbolic Paths

\section{Introduction}

The famous quote of Heraclitus "Nature loves to hide" was described in details by Pierre Hadot in 2008. Hadot in his valuable book gives us many examples how Nature protects Her Secrets. In several situations the enormous research of many generations is strongly needed before the right "recipe" unlocking the true reality can be found.

Conic sections - Circle, Ellipse, Hyperbola, and Parabola - are among the oldest curves, and belong to the Treasure of Geometry. The conic sections seem to have been discovered by Menaechmus and were thoroughly studied by Apollonius of Perga (The Great Geometer) and his scholars. The conics are endowed with numerous beautiful properties, some those properties are shared by the entire family, while some properties are unique and original for each of them. In our research we have to be very careful and patient when we want to apply those properties for the planet orbits. Menaechmus said to Alexander the Great: "O King, for travelling over the country, there are royal roads and roads for common citizens, but in geometry there is one road for all."

The "Conics" of Apollonius of Perga were many times published and inspired all generations of researchers. The astronomer Edmund Halley published one translation in 1710 with a very famous frontispiece. This frontispiece shows a group of shipwrecked survivors on the shore of the island of Rhodes. The Socratic philosopher Aristippus 
found the diagram of the unit hyperbola on the sand: "We have nothing to fear as I see the presence of men". (See Frank Schwetz in 2016).

Johannes Kepler in 1605 made his great breakthrough when he discovered the elliptical path of Mars with the Sun in one focus of that ellipse. Generations of researchers were inspired by this Kepler's ellipse and were searching for properties hidden in those elliptic paths. The great step made Isaac Newton in 1687 when he discovered the locus of radii of curvature of ellipse, hyperbola, and parabola (the evolute of those conic sections) and applied it for the calculation of the centripetal force. W.R. Hamilton in 1847 discovered a very elegant model of the hodograph using the pedal curve with pedal points located in both foci of ellipse (the auxiliary circle of ellipse). However, this classical model of the Kepler's ellipse and Newton's hyperbola could not properly explain the precession of the planets and Albert Einstein in 1915 replaced this classical model with his concept of the elastic spacetime. Our generation has to solve problems with anomalies again because the powerful concept of Albert Einstein has limitations, too. Where should we search our future better models?

A possible chance for further classical development of the Newton's hyperbola is to penetrate more deeply into the secrets of the Newton's hyperbola and to reappear with some new hidden properties overlooked by earlier generations of researchers. Our guiding principle is the existence of the Plato's Realm with invisible mathematical objects that might bring to us some additional information about the visible Newton's hyperbola in the Aristotelian World. In this contribution we have been working with these mathematical objects from the Plato's Realm:

1. Hyperbola properties discovered by Apollonius of Perga - the Great Geometer - and many his scholars.

2. Locus of the radii of curvature (evolute) of hyperbola - Isaac Newton in 1687. A new formula discovered trigonometrically for the radius of curvature of hyperbola.

3. The interplay of the empty focus M (= Menaechmus - the discoverer of hyperbola), the center of the hyperbola A (= Apollonius of Perga - the Great Geometer), and the occupied focus N (= Isaac Newton - the Great Mathematician) together form the MAN Hyperbola with several interesting hidden properties of those hyperbolic paths.

4. Pedal curve with the pedal points in both foci - the auxiliary circle - the newly discovered hodograph of tangent velocities.

5. Pedal curve with the pedal point in the center of that hyperbola - the lemniscate of Bernoulli - the newly discovered hodograph of normal velocities.

6. Torque of the Newton's hyperbola.

7. The auxiliary circle of hyperbola used as the inversion curve to extract the Kepler's Equation for the hyperbolic path. The Lemniscate of Bernoulli for the determination of the third Kepler's law for hyperbolic paths with fictious period of rotation around the radius of curvature.

The experimental analysis of properties of the MAN Hyperbola should reveal if we have found the Arriadne's Thread leading out of the Labyrinth or if we are still lost in the Labyrinth.

(We are aware of the famous quote of Richard Feynman from the year 1962: "There's certain irrationality to any work in gravitation, so it is hard to explain why you do any of it.")

\section{Some Properties of the Unit Hyperbola}

Figure 1 and Figure 2 shows some trigonometric and hyperbolic properties of the unit hyperbola that might be used for the description of motion of planets around the Sun on the hyperbolic orbits. The gudermannian (gdx) provides an important linkage between the circular and hyperbolic functions. The hyperbolic functions were systematically studied by Johann Heinrich Lambert in 1768 and by Christoph Gudermann in 1833.

Table 1 summarizes some relations for the unit hyperbola.

Table 2 compares the circular and hyperbolic functions useful for the evaluation of Newton's hyperbola. 
Table 1. Some properties of the unit hyperbola
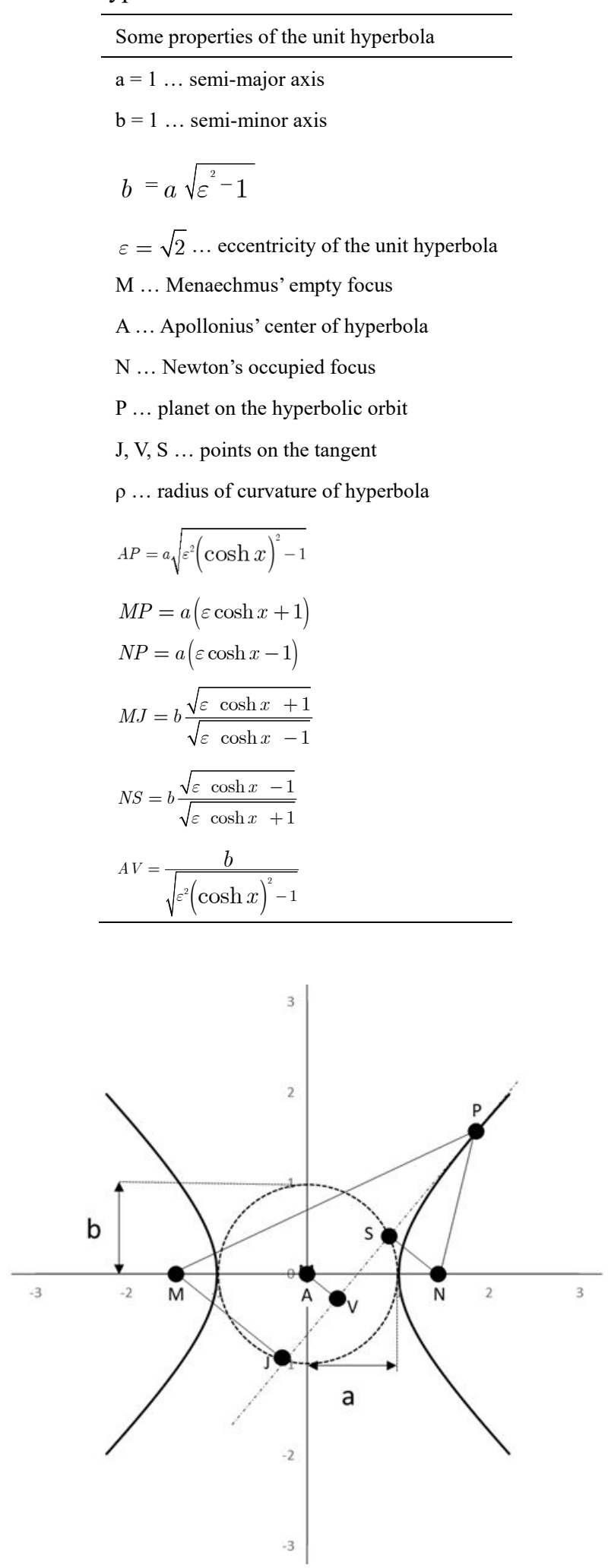

Figure 1. Some relations for the unit hyperbola 


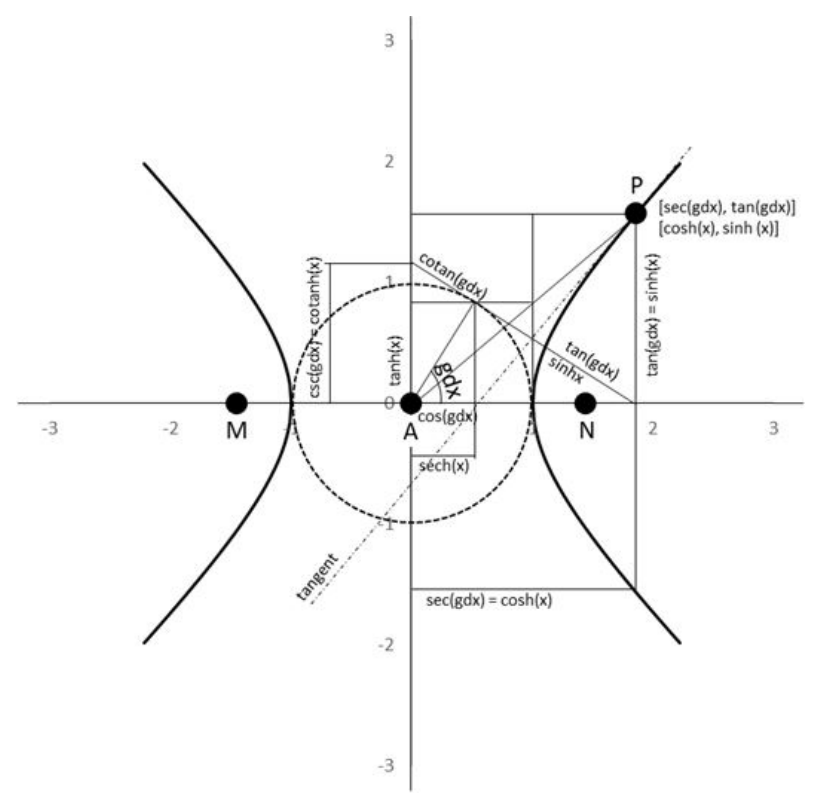

Figure 2. Trigonometric and hyperbolic functions in the unit hyperbola

Table 2. Circular and hyperbolic functions for the unit hyperbola where gdx is the gudermannian of the hyperbolic angle $\mathrm{x}$

\begin{tabular}{l}
$\sin (\operatorname{gdx})=\tanh (\mathrm{x})$ \\
$\cos (\mathrm{gdx})=\operatorname{sech}(\mathrm{x})$ \\
$\tan (\mathrm{gdx})=\sinh (\mathrm{x})$ \\
$\cot (\mathrm{gdx})=\operatorname{csch}(\mathrm{x})$ \\
$\sec (\mathrm{gdx})=\cosh (\mathrm{x})$ \\
$\csc (\mathrm{gdx})=\operatorname{coth}(\mathrm{x})$ \\
\hline
\end{tabular}

\section{Proposed Reflecting and Refracting Properties of Solar and Planet Gravitons}

In this section we will assume that Solar gravitons enter into the internal volume of planets and collide with planet gravitons in four possible scenarios as it was in details described by Jiří Stávek (2018). Gravitons are reflected and refracted similarly as in the case of the Kepler's ellipse. Therefore, we will present here only a schema of reflective and refractive properties of hyperbola in Figure 3.

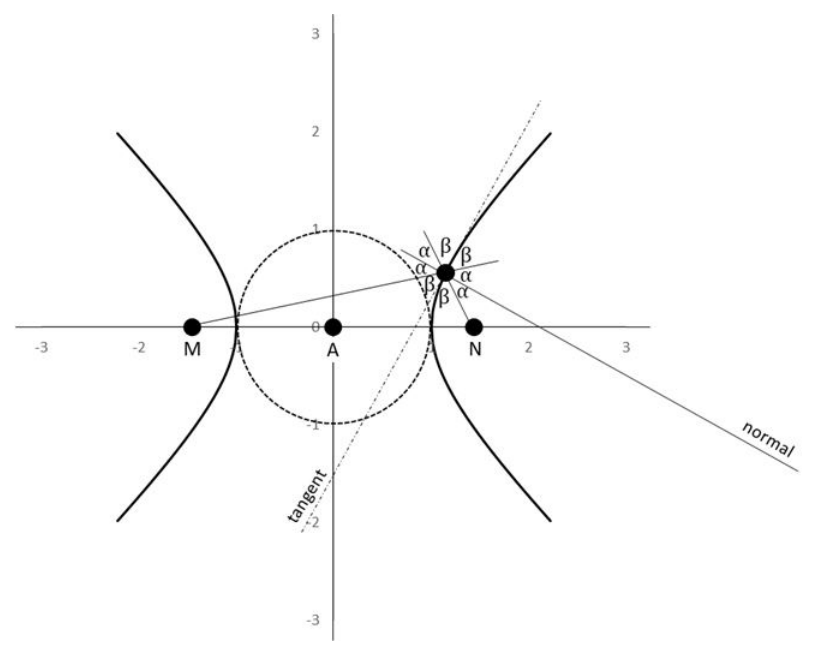

Figure 3. Reflective and refractive properties of hyperbola 


\section{Newton's Hyperbola Observed from the Newton's Evolute}

Newton discovered several important properties hidden in the Kepler's ellipse in his Principia in 1687. For the centripetal force $\mathrm{F}$, he derived formula:

$$
F=m \frac{v_{T}^{2}}{\rho}
$$

where $m$ is the mass of the planet, $v_{T}$ is the tangent velocity of the planet and $\rho$ is the radius of curvature of that ellipse. The locus of radii of curvature is termed as the evolute. This equation opened completely new possibilities in the understanding of the Kepler's ellipse. We will use this procedure for the description of events in Newton's hyperbola.

In the standard procedure both quantities $\mathrm{v}_{\mathrm{T}}$ and $\rho$ are found by the derivation method invented independently by Newton and Leibniz.

We will present here the trigonometric approach to these two quantities $\left(\mathrm{v}_{\mathrm{T}}\right.$ in the next chapter). The radius of curvature of the hyperbola $\rho$ can be derived in the trigonometric way shown in Figure 3. Figure 3 describes an interplay between the normal to the tangent and the line connecting the Sun and orbiting planet.

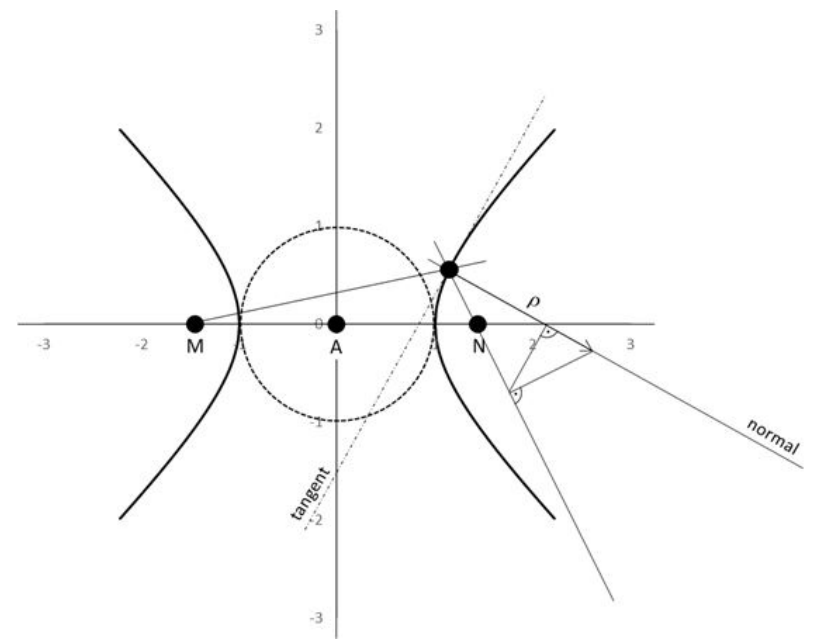

Figure 4. Trigonometric approach to reveal the expression for the radius of curvature $\rho$ of Newton's hyperbola

We have used the deep knowledge of hyperbola properties of Issac Todhunter (1881) and extracted from Figure 3 this "remarkable" expression for the radius of curvature:

$$
\rho=\frac{a^{2}}{b} \times\left(\varepsilon^{2} \cosh ^{2} x-1\right)^{3 / 2}
$$

(The quantities expressed in the trigonometric language are simpler and Nature can talk with us in this trigonometric language that could be depicted in simple Figures without words).

\section{Newton's Hyperbola Observed from the Pedal Curve with Pedal Points in both Foci}

The pedal curve of the Newton's hyperbola is the locus of the feet of the perpendiculars from both foci to the tangent of that hyperbola. In this case the pedal curve is the famous auxiliary circle of the hyperbola.

We were inspired by W. R. Hamilton who in 1847 discovered this concept for the Kepler's ellipse that is known as the hodograph. This approach was several times forgotten and its beauty was several times rediscovered by many researchers. E.g., Richard Feynman in his "Lost lecture" made this concept very well known for our generation.

We can elegantly interpret the quantity of the tangent planet velocity as the perpendicular from the Menaechmus' empty focus to the tangent of Newton's hyperbola. Figure 4 depicts both perpendiculars from the Menaechmus' empty focus and the Newton's occupied focus. The vector of PP' is rotated clockwise by the angle $\pi / 2$. 


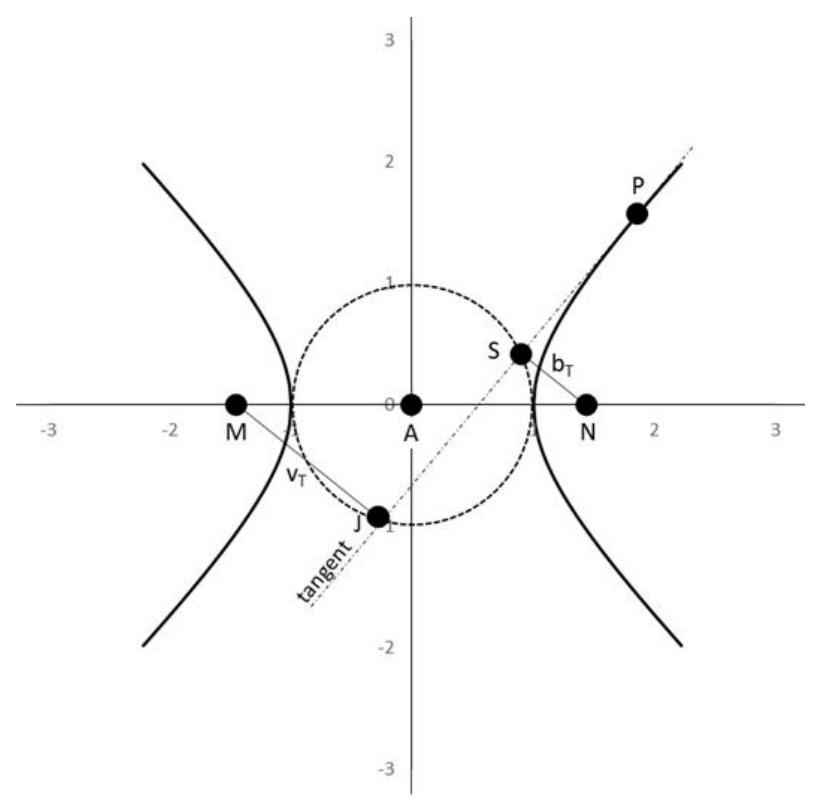

Figure 5. Trigonometric approach to reveal the expression for the tangent velocity $\mathrm{v}_{\mathrm{T}}$ of Newton's hyperbola

We get from Figure 5 the tangent velocity $\mathrm{v}_{\mathrm{T}}$ as:

$$
v_{T}=v_{\infty} \frac{\sqrt{\varepsilon \cosh x+1}}{\sqrt{\varepsilon \cosh x-1}}
$$

where $v_{\infty}$ is the tangent orbital velocity far away from the Sun.

Now, we can test the validity of the Newton's formula expressed in the trigonometric language and compare these trigonometric formulae with formulae obtained in other mathematical languages. A very good inspiration can be found in the valuable book of Arjun Tan (2008).

The famous Newton's formula can be trigonometrically expressed as:

$$
\begin{aligned}
& F=\frac{G M m}{R^{2}}=m \frac{v_{T}^{2}}{\rho} \frac{1}{\cos \alpha}= \\
& =m \frac{v_{\infty}^{2}\left(\frac{\varepsilon \cosh x+1}{\varepsilon \cosh x-1}\right)}{\frac{a^{2}}{b}\left(\varepsilon^{2} \cosh ^{2} x-1\right)^{3 / 2}} \frac{a \sqrt{\varepsilon^{2} \cosh ^{2} x-1}}{b}=\frac{m v_{\alpha}^{2} a}{R^{2}}
\end{aligned}
$$

At the end we have obtained the standard gravitational parameter $\mu$ :

$$
\mu=G M=a v_{\infty}^{2}
$$

where a is the semi-major axis and $v_{\infty}$ is the tangent orbital velocity far away from the Sun.

\section{Newton's Hyperbola Observed from the Lemniscate of Bernoulli as the Pedal Curve of the Newton's Hyperbola}

We want to find a trigonometric expression for the normal velocity $\mathrm{v}_{\mathrm{N}}$ of the planet on the hyperbolic orbit. In this case the MAN Hyperbola shows Her original Feature and Beauty. We cannot use the contrapedal curve as in the case of Kepler's ellipse. We have found a great support from Jacob Bernoulli and His famous Lemniscate of Bernoulli.

The pedal curve of the Newton's hyperbola with the pedal point in the center of the hyperbola is the famous Lemniscate of Bernoulli. 
Figure 5 shows a schema with one pedal point located in the center of the Newton's hyperbola A and the foot point $\mathrm{V}$ is a part of the Lemniscate of Bernoulli. We propose to use the distance AV between the center of the hyperbola $\mathrm{A}$ and the foot point $\mathrm{V}$ as the measure for the normal velocity $\mathrm{v}_{\mathrm{N}}$ :

$$
v_{N}=\frac{v_{\infty}}{\sqrt{\varepsilon^{2} \cosh ^{2} x-1}}
$$

where $\mathrm{V}_{\infty}$ is the tangent orbital velocity far away from the Sun. This is a new hodograph for the normal velocities of planets orbiting in the Newton's hyperbolas.

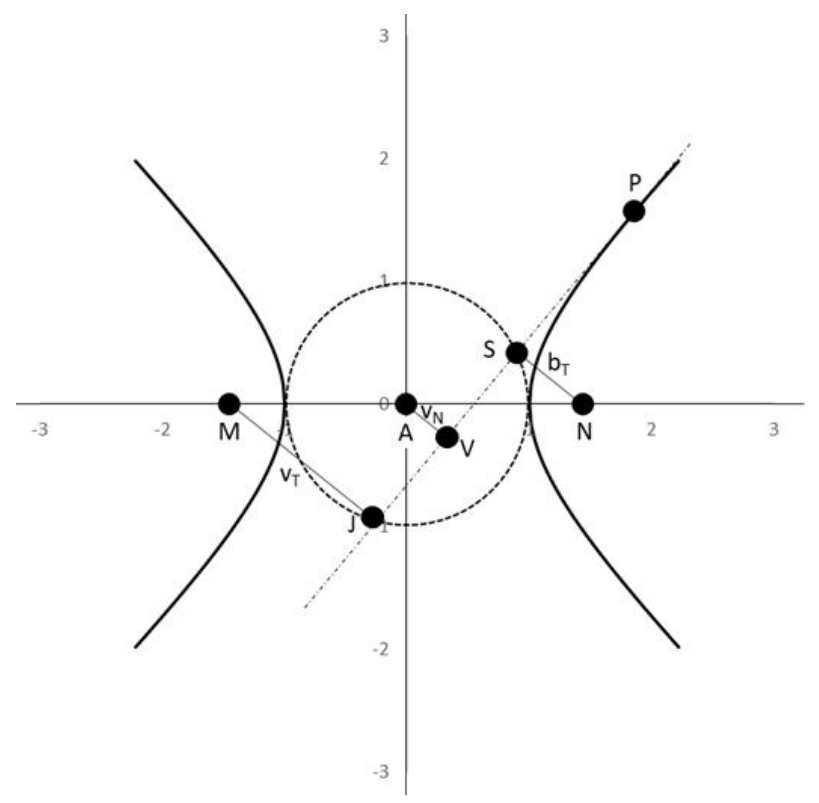

Figure 6. Trigonometric approach to reveal the expression for the normal velocity $\mathrm{v}_{\mathrm{N}}$ for the MAN Hyperbola

\section{Moment of the Tangent Momentum and Moment of the Normal Momentum of the Newton's Hyperbola}

Based on the formulae in Tables 1 and 2 we can evaluate the moment of the tangent momentum $\mathrm{L}_{\mathrm{T}}$ and to introduce a new physical quantity - the moment of the normal momentum $L_{N}$.

The moment of momentum $\mathrm{L}$ is defined as the product of the linear momentum with the length of the moment arm, a line dropped perpendicularly from the origin onto the path of the particle. It is this definition: $\mathrm{L}=$ (length of moment arm) x (linear momentum).

The moment of the tangent momentum $\mathrm{L}_{\mathrm{T}}$ for the Newton's hyperbola is given as:

$$
L_{T}=m v_{T} S N=m v_{\infty} \frac{\sqrt{\varepsilon \cosh x+1}}{\sqrt{\varepsilon \cosh x-1}} b \frac{\sqrt{\varepsilon \cosh x-1}}{\sqrt{\varepsilon \cosh x+1}}=m v_{\infty} b
$$

where $\mathrm{m}$ is the mass of the planet, $\mathrm{v}_{\mathrm{T}}$ the tangent velocity of the planet and $\mathrm{SN}$ is the length of the moment arm (the distance between the Newton's occupied focus and the tangent). The moment of the tangent momentum $\mathrm{L}_{\mathrm{T}}$ is constant during the complete hyperbolic path of the Newton's hyperbola. Therefore, there is no contribution to the torque from this moment of the tangent momentum. This is very well-known experimental fact documented in the existing literature.

The moment of the normal momentum $\mathrm{L}_{\mathrm{N}}$ for the Newton's hyperbola is given as:

$$
\begin{aligned}
& L_{N}=m v_{N} S N=m \frac{v_{\infty}}{\sqrt{\varepsilon^{2} \cosh ^{2} x-1}} b \frac{\sqrt{\varepsilon \cosh x-1}}{\sqrt{\varepsilon \cos E+1}} \\
& =m v_{\infty} b \frac{1}{\varepsilon \cosh x+1}=\frac{m v_{\infty} b a}{M P}
\end{aligned}
$$


where $\mathrm{m}$ is the mass of the planet, $\mathrm{v}_{\mathrm{N}}$ the normal velocity of the planet and $\mathrm{SN}$ is the length of the moment arm (the distance between the Newton's occupied focus and the tangent). The moment of the normal momentum is not constant during the complete path of the Newton's hyperbola - it is dependent on the distance from the Menaechmus empty focus. Therefore, we expect a contribution to the torque of the Kepler's ellipse.

\section{Torque of the Newton's Hyperbola}

Torque is defined mathematically as the rate of the change of the moment of the momentum. As long as the moment of the tangent momentum is constant then there is no net torque applied. However, what about the moment of the normal momentum?

The derivation of the formula for the torque caused by the moment of the normal momentum for the Newton's Hyperbola should be as:

$$
\tau=\frac{d L_{N}}{d t}=\frac{d L_{N}}{d x} \frac{d x}{d t}=\frac{d L_{N}}{d x} \frac{v_{T}}{\rho}=-\frac{m v_{\alpha}^{2} b^{2}}{N P^{2}} \frac{\varepsilon \sinh x}{(\varepsilon \cosh x+1)^{2}}
$$

In this formula we have expressed the rate of change of the hyperbolic angle $\mathrm{x}$ as the ratio of the tangent velocity $\mathrm{v}_{\mathrm{T}}$ of the planet and the curvature $\rho$ in that point of the MAN Hyperbola. The torque $\tau$ depends on the inverse square of the distance of the planet from the Sun and on the value of its hyperbolic angle $\mathrm{x}$. In the first quadrant the planet approaches the Sun and therefore the sign of hyperbolic sine is $(+\operatorname{sinhx})$, in the fourth quadrant the planet travels away from the Sun and the sign of the hyperbolic sinus is (-sinhx).

We expect that this newly discovered quantity $\tau$ - the torque of the Newton's hyperbola - might contribute to the hyperbola precession. There are several very well documented flyby anomalies - discrepancies between current scientific models and the actual increase in the speed observed during a planetary flyby by a spacecraft. One such famous example is the Pioneer anomaly discovered by the great Master John D. Anderson.

We want to pass this concept into the hands of experienced Readers of this Journal. Have we found the Arriadne's Thread leading out of the Labyrinth or are we still lost in the Labyrinth?

Isaac Newton in his Propositions 43-45 of Book I in his Principia derived a formula for the force causing the precession of planets. The astrophysicist Subrahmanyam Chandrasekhar in 1995 in his comments to Principia remarked that this Theorem remained largely unknown and undeveloped for over three centuries. How to describe classically the planet and spacecraft precession?

\section{Torque of the Kepler's Ellipse - Correction of Jiří Stávek 28.10.2018}

Torque is defined mathematically as the rate of the change of the moment of the momentum. As long as the moment of the tangent momentum is constant then there is no net torque applied. However, what about the moment of the normal momentum?

The derivation of the formula for the torque caused by the moment of the normal momentum for the Kepler's ellipse would be as:

$$
\tau=\frac{d L_{N}}{d t}=\frac{d L_{N}}{d E} \frac{d E}{d t}=\frac{d L_{N}}{d E} \frac{v_{T}}{\rho}=\frac{m v_{0}^{2} a b}{R^{2}} \varepsilon^{2} \sin (2 E)
$$

In this formula we have expressed the rate of change of the eccentric anomaly $\mathrm{E}$ as the ratio of the tangent velocity $\mathrm{v}_{\mathrm{T}}$ of the planet and the curvature $\rho$ in that point of the Kepler's ellipse. The torque $\tau$ depends on the inverse square of the distance of the planet from the Sun R and on the value of its eccentric anomaly. In this definition the value $\mathrm{dE} / \mathrm{dt}$ is not dependent on the position of the observer in the Solar System.

\section{Kepler's Equation for the Newton's Hyperbola}

Kepler's Equation (KE) has been in the focus in the modern science for four centuries. This topic passed through hands of numerous great researchers. Peter Colwell (1993) surveyed different mathematical techniques and styles to solve the KE for the Kepler's ellipse - for a given mean anomaly M to find the eccentric anomaly E.

We propose to employ the auxiliary circle of the Newton's hyperbola as the inversion curve for the Newton's hyperbola. This transformation of hyperbola leads into the famous Lemniscate of Bernoulli. We will use the properties of the Lemniscate of Bernoulli for the expression of the fictious angular velocity $\omega$ and the fictious period T to express the third Kepler's law for the hyperbolic orbit. In this case the physical interpretation of the 
period $\mathrm{T}$ is the fictious rotation of the planet around the radius of curvature $\rho$. This situation is depicted by Figures 7 and 8 .

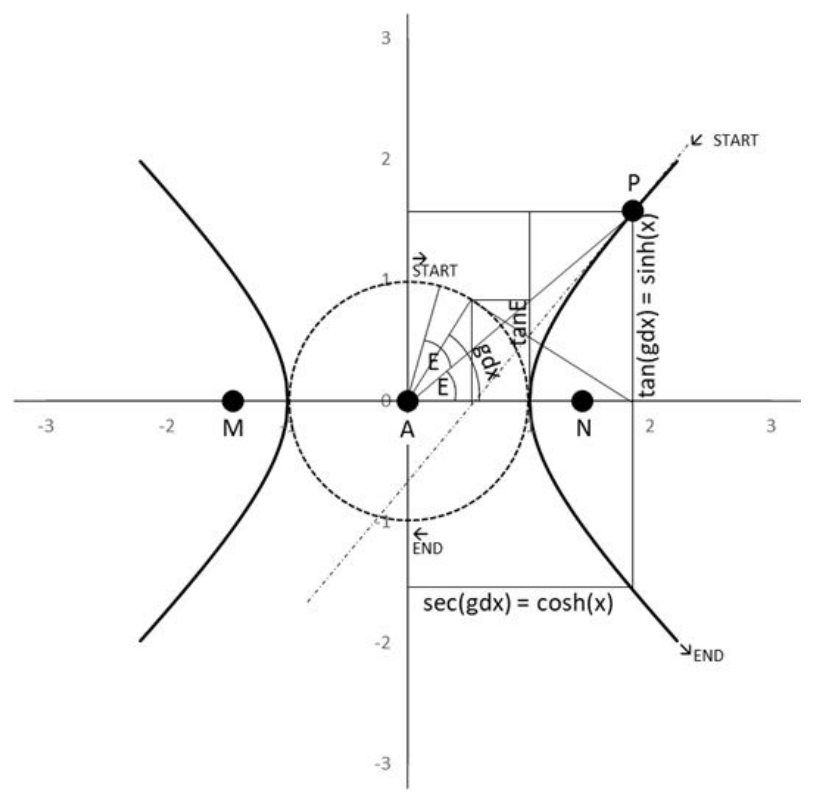

Figure 7. A proposal for the value of the mean anomaly $\mathrm{M}$ for the hyperbolic orbit calculated for the eccentric anomaly $\mathrm{E}(\mathrm{M}=2 \mathrm{E})$

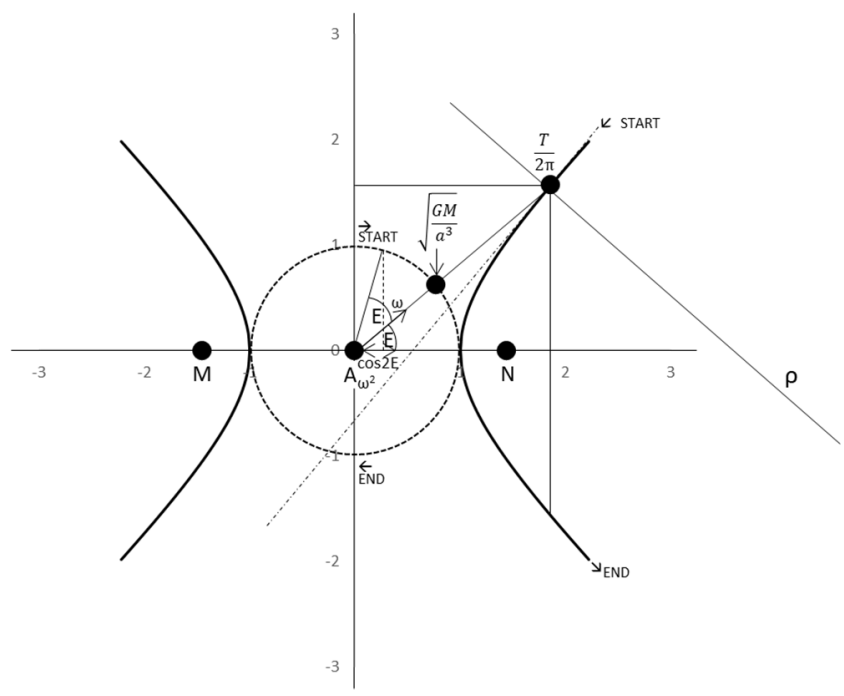

Figure 8. A proposal for the determination of the fictious angular velocity $\omega$ and the fictious period of rotation $\mathrm{T}$ around the radius of curvature $\rho$. The Lemniscate of Bernoulli is the inversion of the Newton's hyperbola around the auxiliary circle

The relations between the mean anomaly $\mathrm{M}$ and the eccentric anomaly E for the Newton's hyperbola might be expressed as:

$$
\tan \frac{M}{2}=\tan E=\tanh x=\frac{\sinh x}{\cosh x}=\frac{\tan (g d x)}{\sec (g d x)}=\sin (g d x)
$$

In 1619 Johannes Kepler published his famous Third Kepler's Law for elliptic orbits. Can we find in 2019 (after four hundred years) an analogy of this Third Kepler's Law for hyperbolic orbits where there is no physical meaning for the period $\mathrm{T}$ ?

We can express the fictious Third Kepler's Law for the Newton's Hyperbola as: 


$$
\left(\frac{T}{2 \pi}\right)^{2}=\frac{a^{3}}{G M} * \frac{1}{\cos (2 E)}=\frac{a^{3}}{G M} * \frac{1}{\cos (M)}
$$

This fictious period $\mathrm{T}$ found using the properties of the Lemniscate of Bernoulli might be an example that the Newton's Hyperbola is surrounded by several hidden curves that reveal to us some new hyperbola properties. Some of those properties have real physical meaning but some of them describe just fictious events.

\section{1. "Antikythera Mechanism" in the Solar System}

We propose to use the very-well known Antikythera Mechanism as an analogy for the visible Newton's hyperbola - a part of our Aristotelian World - connected deeply with invisible curves from the Plato's Realm - Newton's evolute (1687), the Lemniscate of Bernoulli (1694), and the auxiliary circle that expand our knowledge about the visible Newton's hyperbola. Are there some more hidden curves in the Plato's Realm connected to the Newton's hyperbola? How to distinguish the real physical meaning written in those curves from fictious events if both are mathematically correct?

\section{Conclusions}

1. We have presented some quantitative properties of the Newton's hyperbola in Table 1 and Figures 1 and 2.

2. We have studied the interplay of the empty focus of hyperbola M (=Menaechmus) with the center of hyperbola A (=Apollonius of Perga) and the occupied focus of hyperbola N (=Newton) in the MAN Hyperbola.

3. We have discovered a new trigonometric formula for the radius of curvature in the Newton's evolute of the Newton's hyperbola.

3. In the pedal curve with the pedal points in both foci (the auxiliary circle) we have depicted a new hodograph for the determination of tangent velocities of planets on the hyperbolic orbit and the moment of tangent momentum.

4. In the pedal curve with the pedal point in the center of the hyperbola (the Lemniscate of Bernoulli) we have depicted a new hodograph for the determination of normal velocities of planets on the hyperbolic orbit and the moment of normal momentum

5. We have derived the formula for the torque of the Newton's hyperbola.

6. We have corrected the formula for the torque of the Kepler's ellipse in Jiří Stávek (28.10.2018)

7.We have inverted the Newton's hyperbola into the Lemniscate of Bernoulli and proposed the solution of the Kepler's Equation (KE) for the hyperbolic orbit.

8. We have formulated the Third Kepler's Law for the hyperbolic orbit using the fictious period T around the radius of the curvature.

8. Are there some more hidden curves in the Plato's Realm connected to the Newton's hyperbola? How to distinguish the real physical meaning written in those curves from fictious events if both are mathematically correct?

\section{Acknowledgments}

This work was supported by the JP\&FŠ Agency (Contract Number 25g/1963), by the VZ\&MŠ Agency (Contract Number 16000/1989) and by the GMS Agency (Contract Number 69110/1992). JP organized for us the very stimulating seminar on the Feynman's lost lecture (18.12.2018). We were supported by the contract number 28101918/2018. We have found the valuable support on the web site www.wolframalpha.com with the corrections of used formulae.

\section{Conflict of interests}

The authors declare that there is no conflict of interests regarding the publication of this paper.

\section{References}

Abreu, J. L., \& Barot, M. (n. d.). A Geometric Approach to Planetary Motion and Kepler Laws. Retrieved from http://arquimedes.matem.unam.mx/jlabreu/GeomKepler.pdf

Adams, J. (1818). The Elements of the Ellipse Together with the Radii of Curvature Relating to that Curve; and of Centripetal and Centrifugal Forces in Elliptical Orbits. Longman, London. Retrieved from https://books.google.cz/books?id=ckKMj7RIpccC\&pg=PA108\&lpg=PA108\&dq=James+Adams+elements + of + the + ellipse $\&$ source $=$ bl\&ots $=$ XKw8Iyleis\&sig $=$ L2udhh3CosWp9N0Z91PuFamQDHg\&hl $=$ cs\&sa $=$ X\&v 
ed=0ahUKEwi8oJv57ubbAhWEY1AKHTa8AXoQ6AEIJzAA\#v=onepage\&q=James\%20Adams\%20eleme nts $\% 20$ of $\% 20$ the $\% 20$ ellipse \&f $=$ false

Aiton, E. (1969). Kepler's Second Law of Planetary Motion. Isis: A Journal of the History of Science, 60, 75-90.

Antikythera Mechanism. (2018). Retrieved from https://en.wikipedia.org/wiki/Antikythera_mechanism

Auxiliary Circle of Hyperbola. (n. d.). Retrieved from https://www3.ul.ie/ rynnet/swconics/ACT\%27S.htm

Barbour, J. (2014). Kepler and Mach's Principle. In J. Bičák \& T. Ledvinka (Eds.), General Relativity, Cosmology and Astrophysics. Fundamental Theories of Physics, 177. Springer Cham.

Bardini, G., \& Gianella, G. M. (2016). A Historical Walk along the Idea of Curvature, from Newton to Gauss Passing from Euler. International Mathematical Forum, 11, 259-278. Retrieved from http://www.m-hikari.com/imf/imf-2016/5-8-2016/p/bardiniIMF5-8-2016.pdf

Barker, P. (1994). Distance and Velocity in Kepler's Astronomy. Annals of Science, 51, 59-73.

Barnet, J. H. (2004). Enter, Stage Center: The Early Drama of the Hyperbolic Functions, Mathematics Magazine. Retrieved from https://www.maa.org/programs/faculty-and-departments/classroom-capsules-and-notes/ enter-stage-center-the-early-drama-of-the-hyperbolic-functions

Beal, W. O. (1913). A Geometric Interpretation of the Function F in Hyperbolic Orbits Corresponding to that of the Ecceentric Anomaly E in Elliptic Orbits. The American Mathematical Monthly, 20, 52-56.

Beckmann, B. (2006). Feymann Says "Newton Implies Kepler, No Calculus Needed". The Journal of Symbolic Geometry, 1, 57-72. Retrieved from http://ceadserv1.nku.edu/longa/classes/calculus_resources/docs/kep.pdf

Beech, M. (2005). On Ptolemy's Equant, Kepler's Second Law, and the Non-existent “Empty-Focus” Cometarium. Journal of the Royal Society of Canada, 120-123.

Besant, W. H. (2009). Conic Sections: Treated Geometrically (9th ed.). Merchant Books. ISBN-10: 1603862579. Retrieved from https://www.gutenberg.org/files/29913/29913-pdf.pdf.

Bičák, J., \& Ledvinka, T. (Eds.) (2014). General Relativity, Cosmology, and Astrophysics. Perspectives 100 years after Einstein's stay in Prague. Springer. ISBN-10: 3319063480.

Blaschke, P. (2017). Pedal Coordinates, Dark Kepler and Other Forces Problems. Arxiv: 1704.00897v1.

Blåsjö, V. (2018). Mathematicians Versus Philosophers in Recent Work on Mathematical Beauty. Journal of Humanstic Mathematics, 8, 414-431. Retrieved from https://scholarship.claremont.edu/cgi/viewcontent.cgi? referer=https://www.google.com/\&httpsredir=1\&article=1380\&context=jhm

Böhm, J. (2016). Wonderful World of Pedal Curves. Retrieved from http://rfdz.ph-noe.ac.at/fileadmin/ Mathematik_Uploads/ACDCA/TIME2016/Boehm_Pedals_.pdf

Brackenridge, J. B. (1996). The Key to Newton's Dynamics: The Kepler Problem and the Principia. Berkeley: University of California Press. ISBN: 978-0520202177.

Bruneau, O. (2015). ICT and History of Mathematics: The Case of the Pedal Curves from 17th Century to 19th Century. Retrieved from https://hal.archives-ouvertes.fr/hal-01179909/document

Cavalieri, B. (1643). Trigonometria plana, et sphcerica, linearis, \& logarithmica. Frontispiece. Retrieved from http://hldigital.lindahall.org/cdm/ref/collection/emblematic/id/1365

Chandrasekhar, S. (1995). Newton's Principia for the Common Reader. Oxford University Press, Oxford, ISBN 978-0-19-852675-9.

Cohen, I. B. (1999). A Guide to Newton's Principia. The Principia: The Mathematical Principles of Natural Philosophy. Berkeley, CA, University California Press. ISBN: 978-0-520-08816-0.

Colwell, P. (1993). Solving Kepler's Equation Over Three Centuries. Willmann-Bell. Inc. Richmond. ISBN 0-943396-40-9.

Darrigol, O. (2012). A History of Optics. From Greek Antiquity to the Nineteenth Century. Oxford University Press. Oxford. ISBN-10: 0199644373.

Derbes, D. (2001). Reinventing the wheel: Hodographic solutions to the Kepler problems. Am. J. Phys., 69, 481-489.

Donahue, W. (1994). Kepler's Invention of the Second Planetary Law. British Journal fort he History of Science, 27, 89-102. 
Einstein, A. (1918). Prinzipielles zur Allgemeinen Relativitätstheorie. Annalen der Physik, 360, 241.

Feynman, R. F. (1963). The Character of Physical Law, Part 1, The Law of Gravitation ("How should angels generate the Kepler ellipse?"). Retrieved from https://www.youtube.com/watch?v=j3mhkYbznBk

Flyby Anomaly. (n. d.). Retrieved from https://en.wikipedia.org/wiki/Flyby_anomaly

Fried, M., \& Unguru, S. (2001). Apollonius of Perga's Conica: Text, Context, Subtext. Mnemosyne, Bibliotheca Classic. ISBN-10: 9004119779.

Gant, de F. (1995). Force and Geometry in Newton's Principia. Princeton University Press, Princeton. ISBN 0-691-03367-6.

Gingerich, O. (1973). From Copernicus to Kepler: Heliocentrism as Model and as Reality. Proceedings of the American Philosophical Society, 117, 513-522.

Glaser, G., Stachel, H., \& Odehnal, B. (2016). The Universe of Conics: From Ancient Greeks to 21st Century Developments. Springer Spektrum. ISBN-10: 36622454491.

Goodstein, D., \& Goodstein, J. R. (2000). Feynman's Lost Lecture. W. W. Norton \& Company. ISBN-10: 0393319954.

Gudermannian Function. (n. d.a). Retrieved from http://mathworld.wolfram.com/Gudermannian.html

Gudermannian Function. (n. d.b). Retrieved from https://en.wikipedia.org/wiki/Gudermannian_function

Guicciardini, N. (2003). Reading the Principia: The Debate on Newton's Mathematical Methods for Natural Philosophy from 1687 to 1736. Cambridge University Press, Cambridge. ISBN-10: 0521544033.

Hadot, P. (2008). The Veil of Isis: An Essay on the History of the Idea of Nature. Belknap Press. ISBN-10: 0674030494.

Hamilton, W. R. (1847). The Hodograph, or a New Method of Expressing in Symbolical Language the Newtonian Law of Attraction. Proceedings of the Royal Irish Academy, 3, 344-353. Retrieved from https://www.emis.de/classics/Hamilton/Hodo.pdf

Hatfield, B. (Ed.) (2018). Feynman Lectures on Gravity. CRC Press. ISBN 13: 978-0-8133-4038-8 (pbk).

Hawking, S. W., \& Israel, W. (Eds.) (1989). Three Hundred Years of Gravitation. Cambridge University Press, Cambridge. ISBN-10: 0521379768.

Heat, T. L. (2015). Apollonius of Perga: Treatise on Conic Sections. Carruthers Press. ISBN-10: 1446021262.

Horský, Z. (1980). Kepler in Prague. Mír. Prague. 601/22/85.5 (In Czech).

Hsiang, W. Y., \& Straume, E. (2014). Revisiting the mathematical synthesis of the laws of Kepler and Galileo leading to Newton's law of universal gravitation. Arxiv: 1408.6758v1.

Huygens, Ch. (1659) On Centrifugal Force. (De Vi Centrifuga). Translated by M.S. Mahonay. Retrieved from https://www.princeton.edu/ hos/mike/texts/huygens/centriforce/huyforce.htm

Hyperbola Properties. (n. d.a). Retrieved from http://mathworld.wolfram.com/Hyperbola.html

Hyperbola Properties. (n. d.b). Retrieved from https://en.wikipedia.org/wiki/Hyperbola

Hyperbola Properties. (n. d.c). Retrieved from https://www3.ul.ie/ rynnet/swconics/HP\%27s.htm

Hyperbolic Angle. (n. d.). Retrieved from https://en.wikipedia.org/wiki/Hyperbolic_angle

Hyperbolic Functions. (n. d.a). Retrieved from https://de.wikipedia.org/wiki/Sinus_hyperbolicus_und_Kosinus_ hyperbolicus

Hyperbolic Functions. (n. d.b). Retrieved from https://en.wikipedia.org/wiki/Hyperbolic_function

Hyperbolic Trajectory. (n. d.). Retrieved from https://en.wikipedia.org/wiki/Hyperbolic_trajectory

Kawarabuki, H., \& Nakazato, H. (2013). Another Question on Kepler's so Called Distance Law. Int. Math. Forum, 8, 59-63. Retrieved from https://pdfs.semanticscholar.org/3d20/aa16678717a8ceb1062056a37de0036 5faa7.pdf

Kepler, J. (1609). Astronomia Nova. Translated by Max Casper. Marix Verlag. ISBN-10:3865390145.

Kepler, J. (1619). Harmony of Worlds. Translated by Ch. G. Wallis, Ecyclopædica Britannica, Chicago.

Kepler, J. (1627). Tabulace Rudolphinace. Retrieved from https://bibdig.museogalileo.it/Teca/Viewer?an=334726 
Köller, J. (2016). Fusspunktkurven und Gegenfusspunktkurven. Retrieved from http://www.mathematische-bast eleien.de/fusspunktkurve.htm

Laird, W. R., \& Roux, S. (Eds.) (2008). Mechanics and Natural Philosophy before the Scientific Revolution. Springer. ISBN 978-90-481-7491-1.

Lämmerzahl, C. (2008). The Pioneer Anomaly or Do We Really Understand the Physics Within the Solar System? Retrieved from https://www.zarm.uni-bremen.de/fileadmin/images/laemmerzahlDatei/Pioneer_Utrecht.pdf

Lemniscate of Bernoulli. (n. d.a). Retrieve from http://mathcurve.com/courbes2d.gb/lemniscate/lemniscate.shtml

Lemniscate of Bernoulli. (n. d.b). Retrieved from http://mathworld.wolfram.com/Lemniscate.html

Lemniscate of Bernoulli. (n. d.c). Retrieved from https://en.wikipedia.org/wiki/Lemniscate_of_Bernoulli

Lockwood, E. H. (1961). A Book of Curves. Cambridge University Press. Retrieved from http://www.aproged.pt/biblioteca/ABookofCurvesLockwood.pdf

Maor, E., \& Jost, E. (2014). Beautiful Geometry. Princeton University Press, Princeton. ISBN-13:978-0-691-15099-4.

Menaechmus. (n. d.). Retrieved from https://en.wikipedia.org/wiki/Menaechmus

Miller, D. M. (2008). "O Male Factum": Rectilinearity and Kepler's Discovery of the Ellipse. Journal for the History of Astronomy, 39, 43-63. Retrieved from http://adsbit.harvard.edu/cgi-bin/nph-iarticle query?2008JHA....39...43M\&defaultprint=YES\&filetype $=$. .pdf

Nauenberg, M. (2018). Visiting Newton's Atelier before the Principia, 1679-1684. Arxiv: 1805.06871v.

Newton, I. (1687). The Principia. Mathematical Principles of Natural Philosophy. Translated by I.B. Cohen and A. Whitman. University California Press, Berkeley. ISBN 978-0-520-08816-0.

Papaspirou, P., \& Moussas, X. (2013). The Hellenistic and Alexandrian Influences on Johannes' Kepler Work. Retrieved from https://pdfs.semanticscholar.org/fc7f/ab1980955f87e2a53622ceaa0c1ee75e4e60.pdf?_ga=2. 199516460.368964894.1539953979-1077201457.1539953979

Pedal Curves Properties. (2018). Retrieved from https://en.wikipedia.org/wiki/Pedal_curve

Pioneer Anomaly. (n. d.). Retrieved from https://en.wikipedia.org/wiki/Pioneer_anomaly

Podolský, J. (2018, December 20). Feynman's Lost Lecture (In Czech). Retrieved from https://www.youtube.com/watch?v=Un0MOt7o6R8

Roveli, C. (2018). Physics Needs Philosophy. Philosophy Needs Physics. Found. Phys., 48, 481-491. Retrieved from https://arxiv.org/ftp/arxiv/papers/1805/1805.10602.pdf

Russell, J. L. (1964). Kepler's Laws of Planetary Motion: 1609 - 1666. The British Journal for the History of Science, 2, 1-24.

Rynne, S. (2006). Curvature of the Ellipse. Retrieved from https://www3.ul.ie/ rynnet/swconics/E-COC.htm

Sethuraman, J. (2018). Gudermannian - Kepler Equations of Planetary Motion. Retrieved from https://www.academia.edu/3832741/Gudermannian_-_Kepler_Equations_of_Planetary_Motion

Stávek, J. (2018a). Kepler's Ellipse Generated by the Trigonometrically Organized Gravitons. Appl. Phys. Res., 10, 26-37.

Stávek, J. (2018b). Kepler's Ellipse Observed from Newton's Evolute (1687), Horrebow's Circle (1717), Hamilton's Pedal Curve (1847), and Two Contrapedal Curves (28.10.2018). App. Phys. Res., 10, 90-101.

Strutz, Ch. (2001a). Über das Latus Rectum Principalis in Newton's Himmelmechanik. Retrieved from http://www.schulphysik.de/strutz/latrect1.pdf

Strutz, Ch. (2001b). Von Apollonius zur Himmelsmechanik. Retrieved from http://www.schulphysik.de/ strutz/gravit2.pdf

Sugimoto, T. (2009). How to Present the Heart of Newton's Principia to the Layperson: A Primer on the Conic Sections without Apollonius of Perga. Symmetry: Culture and Science, 20, 113-144. Retrieved from http://www.is.kanagawa-u.ac.jp/overview/doc/Symmetry_Sugimoto_2009.pdf

Suzuki, M. S., \& Suzuki, I. S. (2015). Hodographic Solutions to the Kepler's problem. Retrieved from https://www.researchgate.net/profile/Masatsugu_Suzuki/publication/271763295_Hodographic_Solutions_to 
_the_Kepler\%27s_Problem_Masatsugu_Sei_Suzuki_and_Itsuko_S_Suzuki/links/54d0d2720cf298d656691 a67/Hodographic-Solutions-to-the-Keplers-Problem-Masatsugu-Sei-Suzuki-and-Itsuko-S-Suzuki.pdf

Švejda, A. (2004). Kepler and Prague. National Technical Museum. ISBN: 80-7037-130-7.

Swetz, F. (2016). Mathematical Treasure: Halley's Conics of Apollonius. Convergence. Retrieved from https://www.maa.org/press/periodicals/convergence/mathematical-treasure-halleys-conics-of-apollonius

Tan, A. (2008). Theory of Orbital Motion. World Scientific Publishing Co., Singapore. ISBN-10: 981-270-911-8.

Todhunter, I. (1881). A Treatise on Plane Co-ordinate Geometry as Applied to the Straight Line and the Conic Sections. MacMillan and Co. London. Retrieved from https://projecteuclid.org/euclid.chmm/1263315392

Wolfram, S. (2018). The History and Future of Special Functions. Retrieved from https://www.stephenwolfram.com/publications/history-future-special-functions/

Yates, R. C. (1974). Curves and their Properties. National Council of Teachers of Mathematics, ISBN: 10-087353039X.

Zeuthen, H. G. (1896). Die Lehre von den Kegelschnitten im Altertum. Kopenhagen, A.F. Höst \& Son. Retrieved from https://ia800208.us.archive.org/20/items/dielehrevondenk00zeutgoog/dielehrevondenk00zeutgoog.pdf

\section{Copyrights}

Copyright for this article is retained by the author(s), with first publication rights granted to the journal.

This is an open-access article distributed under the terms and conditions of the Creative Commons Attribution license (http://creativecommons.org/licenses/by/4.0/). 